\title{
A histerectomia simples realizada no menacme e a densidade mineral óssea da mulher na pós-menopausa
}

\author{
Pre-menopausal simple hysterectomy and \\ post-menopausal female bone mineral density
}

João Daniel Hobeika ${ }^{1}$

Aarão Mendes Pinto Neto 1

Lúcia Helena Simões da Costa Paiva 1

Adriana Orcesi Pedro 1

Edson Zangiacomi Martinez 1

\footnotetext{
1 Departamento de Tocoginecologia, Faculdade de Ciências Médicas, Universidade Estadual de Campinas. Rua Alexander Fleming 101, Campinas, $S P$ 13083-970, Brasil. jhobeika@cosmo.com.br
}

\begin{abstract}
This study aimed to evaluate the bone mineral density (BMD) of post-menopausal women with previous pre-menopausal hysterectomy including bilateral ovarian conservation compared to a group of non-hysterectomized women with natural menopause. Data from a cross-sectional study of 30 pre-menopausally hysterectomized women evaluated in the postmenopause were compared with 102 naturally post-menopausal women, analyzing their respective bone densitometry, measuring the femoral and lumbar spinal BMD. Multiple regression analysis of the 132 women showed that age and body mass index (BMI) were heavily associated with femoral and lumbar spinal BMD, BMI directly associated, and age inversely associated with $B M D$. In addition, 30 hysterectomized women were matched by age and BMI to the 30 non-hysterectomized women, and bone densitometry did not show significant differences in BMD. These findings suggest that pre-menopausal hysterectomy with bilateral ovarian conservation does not appear to cause an additional reduction in bone mass when evaluated in the post-menopausal phase.
\end{abstract}

Key words Densitometry; Ovary; Osteoporosis; Menopause; Hysterectomy

Resumo O objetivo foi avaliar a densidade mineral óssea de mulheres na pós-menopausa, com o antecedente de histerectomia com conservação ovariana bilateral, realizada no menacme, comparadas a um grupo de mulheres com menopausa natural, não histerectomizadas. Tratouse de um estudo de corte transversal, com trinta mulheres na pós-menopausa e histerectomizadas no menacme e 102 mulheres menopausadas naturalmente, comparando-se as respectivas densitometrias ósseas, em nível do fêmur e coluna lombar. A análise mostrou que as variáveis idade e índice de massa corporal estavam fortemente associadas à densidade mineral óssea do fêmur e da coluna lombar, sendo o índice de massa corporal diretamente e a idade inversamente associados. Posteriormente, as trinta mulheres histerectomizadas, foram pareadas por idade e indice de massa corporal, com trinta mulheres não-histerectomizadas, não apresentando diferenças estatisticamente significantes da densidade mineral óssea. Esses resultados sugerem que a histerectomia com conservação ovariana bilateral, realizada em mulheres no menacme, não parece ocasionar redução adicional da massa óssea, quando avaliadas na pós-menopausa.

Palavras-chave Densitometria; Ovários; Osteoporose; Menopausa; Histerectomia 


\section{Introdução}

A histerectomia é uma das cirurgias ginecológicas mais freqüentemente realizadas em mulheres adultas, nos países desenvolvidos. Entre 1988 e 1990, cerca de 1,7 milhões de mulheres foram histerectomizadas nos Estados Unidos (Wilcox et al., 1994). No Brasil, em 1996, foram realizadas 77.804 histerectomias financiadas pelo Sistema Único de Saúde (SUS) (O’Dwyer, 1998).

As causas mais freqüentes para essa intervenção, no menacme e na pré-menopausa, são os miomas uterinos, as irregularidades menstruais rebeldes ao tratamento clínico e a endometriose. Na pós-menopausa essas indicações diminuem de freqüência, enquanto aumentam as indicações cirúrgicas por prolapso uterino e pelas patologias ginecológicas malignas. As principais complicações da histerectomia são: hemorragia intra e pós-operatória, infecção e deiscência da parede abdominal, infecção urinária e pneumonia pós-cirúrgica, lesões dos órgãos adjacentes como ureter, bexiga e intestino e a doença tromboembólica. Além dessas, devem ser citadas algumas complicações tardias da histerectomia, tais como os distúrbios sexuais, as disfunções do trato urinário inferior e a constipação intestinal, essa última devido a aderências pélvicas pós-histerectomia.

Nas histerectomias realizadas em mulheres na pré-menopausa, tenta-se, sempre que possível, preservar os ovários, acreditando estar prevenindo a menopausa cirúrgica e suas conseqüências. Embora se considere que a histerectomia simples não afete a função ovariana, evidências sugerem que mais de $34 \%$ das mulheres histerectomizadas irão desenvolver um certo grau de falência ovariana dentro de dois anos após a cirurgia, mesmo com a conservação desses órgãos (Quinn et al., 1994).

Souza et al. (1986) avaliaram a histologia ovariana de 25 mulheres, imediatamente antes da histerectomia, e após um ano da mesma. $\mathrm{O}$ estudo histológico mostrou diminuição da reserva folicular ovariana, um ano após a histerectomia.

A idade de ocorrência da falência ovariana pós-histerectomia foi avaliada por Siddle et al. (1987) em noventa mulheres histerectomizadas com conservação dos ovários. Observaram que no grupo das mulheres histerectomizadas, a média de idade à falência ovariana era de 45,4 anos, enquanto que no grupo controle ocorreu aos 49,5 anos.

A dosagem das gonadotrofinas e do estradiol sangüíneo de 110 mulheres histerectomizadas com conservação ovariana, entre 38 e 48 anos, foram estudadas por Kaiser et al. (1989), utilizando como grupo controle, mulheres sem cirurgia pélvica. Observaram-se diferenças estatisticamente significativas, com maiores níveis de FSH e LH, e menores de estradiol sérico, no grupo de mulheres histerectomizadas.

Por outro lado, Lima et al. (1996) estudaram a função ovariana pós-histerectomia em vinte mulheres, com idade entre 20 e 40 anos, e verificaram que nenhuma das pacientes apresentou alteração que pudesse denunciar um quadro de falência ovariana.

Tem-se descrito a "Síndrome pós-histerectomia", na qual alguns sinais e sintomas climatéricos apareceriam precocemente, fato esse atribuído à redução da produção ovariana de estrogênios. A causa não é totalmente esclarecida, mas parece estar relacionada a uma diminuição ou alteração na circulação sangüínea ovariana.

Se esse comprometimento pode afetar a função ovariana e até levar a ocorrência da menopausa mais precocemente, devemos preocupar-nos com as conseqüências deste déficit hormonal precoce. Dentre as preocupações, destaca-se a osteoporose relacionada à pósmenopausa, que geralmente ocorre entre os 50 e 60 anos. A osteoporose é uma doença de etiologia multifatorial, mas é bem conhecido que a deficiência estrogênica da pós-menopausa é o principal fator desencadeante. A disfunção ovariana e a conseqüente diminuição estrogênica são responsáveis pelo rápido aumento da reabsorção óssea, levando a uma diminuição da densidade mineral óssea com perda acelerada do osso predominantemente trabecular e maior incidência de fraturas.

Assim, realizamos este estudo com o objetivo de avaliar a densidade mineral óssea de mulheres na pós-menopausa, com o antecedente cirúrgico de histerectomia com conservação ovariana bilateral, realizada no menacme, comparadas a um grupo de mulheres com menopausa natural, não histerectomizadas.

\section{Pacientes e métodos}

As mulheres foram selecionadas no Ambulatório de Menopausa do Departamento de Tocoginecologia (DTG), do Centro de Atenção Integral à Saúde da Mulher (CAISM), da Universidade Estadual de Campinas (UNICAMP), no período entre setembro de 1997 a fevereiro de 1999, respeitando-se os critérios de admissão no estudo.

Foram avaliadas algumas características clínicas, gineco-obstétricas e a densitometria óssea (Lunar DPX) de trinta mulheres na pós-me- 
nopausa, submetidas à histerectomia com conservação ovariana bilateral, realizada no menacme (Grupo "Histerectomizadas") e comparadas a 102 mulheres com menopausa natural (Grupo “Não-histerectomizadas”). Para o grupo "histerectomizadas", foram incluídas mulheres com o antecedente de histerectomia com conservação ovariana bilateral, idade entre 45 e 64 anos, cor branca ou parda e com dosagem de FSH plasmático $\geq 30 \mathrm{UI} / \mathrm{ml}$, confirmando a menopausa. Para o grupo "não-histerectomizadas" incluímos mulheres com útero e ovários preservados, com a mesma faixa etária e cor da pele e que apresentassem amenorréia maior que 12 meses.

Excluímos as mulheres que apresentassem doenças como o diabetes mellitus e as alterações tireoideanas, e também as usuárias de medicamentos que sabidamente alteram o metabolismo ósseo. Também excluímos as com antecedente de radioterapia ou quimioterapia prévia, as tabagistas de mais de 20 cigarros/dia, as etilistas de mais de dois copos de bebida alcoólica/dia e finalmente, as mulheres que apresentassem um índice de massa corporal (IMC) $<20 \mathrm{~kg} / \mathrm{m}^{2} \mathrm{ou}>30 \mathrm{~kg} / \mathrm{m}^{2}$.

Todas as mulheres selecionadas durante consulta de rotina responderam a um questionário contendo os dados necessários ao estudo. Nesse mesmo dia, foi colhida amostra de sangue das mulheres histerectomizadas para dosagem do FSH plasmático. Posteriormente, todas as pacientes selecionadas foram encaminhadas ao Setor de Medicina Nuclear do Hospital das Clínicas da UNICAMP, para realização da densitometria óssea do fêmur e da coluna lombar pela técnica DEXA (dual energy $x$-ray absorptiometry) utilizando-se um equipamento Lunar DPX.

A variável independente estudada foi a histerectomia com conservação ovariana bilateral, enquanto que a variável dependente foi a densidade mineral óssea, sendo expressa em valores absolutos em $\mathrm{g} / \mathrm{cm}^{2}$ e valores relativos em T-score (adulto jovem). Segundo os critérios internacionais, os resultados da densitometria óssea são classificados em: normal (valores de T-score maiores ou iguais a - 1 desviopadrão em relação à média do adulto jovem), osteopenia (entre - 1 densidade padrão (DP) e $-2,5 \mathrm{DP}$ ) e osteoporose (menor ou igual a -2,5DP). Neste estudo, esta variável foi agrupada em apenas duas categorias: normal (valores de Tscore maiores ou iguais a -1DP) ou alterado (valores de T-score abaixo de -1DP).

Como as variáveis de controle foram analisadas: idade, índice de massa corporal, cor da pele, escolaridade, tabagismo, idade à menar- ca, paridade, antecedente de laqueadura tubária, tempo de amenorréia, tempo de histerectomia e o tempo de sintomas climatéricos.

Inicialmente foram elaboradas tabelas descritivas, utilizando-se freqüência, média e desvio-padrão, sendo a comparação das médias realizadas através do teste $\mathrm{t}$ de Student. Em situações onde os valores tivessem uma distribuição não-normal, não permitindo comparar médias, utilizou-se o teste não paramétrico de Mann-Whitney. Para a comparação das freqüências, foram utilizados os testes exato de Fisher ou qui-quadrado de Pearson.

Numa segunda etapa, utilizou-se a regressão linear múltipla para identificar quais as variáveis que poderiam estar associadas às alterações da densidade mineral óssea do fêmur e da coluna lombar, utilizando-se o método de seleção stepwise. Para a análise de regressão linear múltipla, foram estudadas as variáveis: idade, cor, escolaridade, índice de massa corporal, tabagismo, menarca, paridade e os antecedentes de laqueadura tubária e de histerectomia simples.

Posteriormente, foi realizado um pareamento segundo os fatores que se apresentaram significativamente associados à densidade óssea na regressão múltipla, tanto em nível do fêmur como da coluna lombar, para controlar as principais variáveis confundidoras. Escolheuse a primeira mulher da lista do grupo não-histerectomizadas que apresentasse resultados semelhantes das variáveis do pareamento com a mulher histerectomizada em questão. Ao final, apresentamos trinta mulheres histerectomizadas pareadas com trinta mulheres nãohisterectomizadas.

Para a comparação das médias da densidade mineral óssea (DMO) em $\mathrm{g} / \mathrm{cm}^{2}$ e dos valores relativos em T-score, em nível do fêmur, foi utilizado o teste de Bonferroni, que faz uma comparação múltipla dos três locais analisados. Em nível da coluna lombar utilizou-se o teste t de Student para amostras independentes. A análise da distribuição segundo categorias de T-score normal ou alterada, de acordo com os locais analisados, foi realizada utilizando-se o teste quiquadrado de Pearson. Esta pesquisa foi aprovada pela Comissão de Pesquisa do CAISM/UNICAMP e Comitê de Ética em Pesquisa da Faculdade de Ciências Médicas/UNICAMP.

\section{Resultados}

A Tabela 1 apresenta algumas características das mulheres estudadas. Não houve diferenças estatisticamente significativas em relação à ida- 
de, índice de massa corporal, escolaridade e porcentagem de mulheres brancas e tabagistas nos dois grupos.

Em relação aos antecedentes gineco-obstétricos, a comparação entre os grupos não mostrou diferenças significativas em relação à idade na menarca e ao antecedente de laqueadura tubária. Não houve diferença significativa na média da paridade entre os grupos, entretanto, observou-se diferença significativa quando a paridade foi dicotomizada em nulíparas e multíparas. Nas mulheres não-histerectomizadas, o tempo médio de amenorréia foi de 3,9 anos. O tempo médio dos sintomas climatéricos nas mulheres histerectomizadas foi de 4,2 anos. As mulheres do grupo "histerectomizadas" tinham sido submetidas a essa cirurgia em média há 12 anos, variando entre 1 e 25 anos. A média de idade dessas mulheres, na época da histerectomia, foi de 41 anos (Tabela 2).

$\mathrm{Na}$ análise de regressão linear múltipla, as variáveis significativamente associadas à DMO do fêmur e da coluna lombar, foram o índice de massa corporal e a idade. O IMC mostrou ter uma associação direta, ou seja, quanto maior o IMC, maior a DMO nestes locais. Em relação à idade, houve uma associação inversa com a DMO do fêmur e da coluna lombar (Tabela 3).

Na coluna lombar, as variáveis cor parda e escolaridade também mostraram associação

Tabela 1

Características das mulheres histerectomizadas e não-histerectomizadas.

\begin{tabular}{|c|c|c|c|}
\hline Variáveis & $\begin{array}{l}\text { Histerectomizadas } \\
\qquad(n=30)\end{array}$ & $\begin{array}{l}\text { Não-histerectomizadas } \\
\qquad(\mathrm{n}=102)\end{array}$ & $\mathbf{P}$ \\
\hline Idade (anos)* & $53,1 \pm 5,1$ & $52,6 \pm 4,0$ & 0,611 \\
\hline Índice de Massa Corporal $\left(\mathrm{kg} / \mathrm{m}^{2}\right)^{*}$ & $25,8 \pm 3,2$ & $26,4 \pm 3,6$ & 0,411 \\
\hline Escolaridade (anos)* & $4,6 \pm 4,3$ & $4,3 \pm 3,5$ & 0,972 \\
\hline Brancas (\%) & 83,3 & 93,1 & 0,143 \\
\hline Tabagistas (\%) & 23,3 & 13,7 & 0,263 \\
\hline
\end{tabular}

* Média \pm desvio-padrão.

1 Teste $t$ de Student para comparação de médias.

2 Teste não paramétrico de Mann-Whitney.

3 Teste exato de Fisher.

Tabela 2

Características gineco-obstétricas das mulheres histerectomizadas e não-histerectomizadas.

\begin{tabular}{|c|c|c|c|}
\hline Variáveis & $\begin{array}{l}\text { Histerectomizadas } \\
\qquad(n=30)\end{array}$ & $\begin{array}{l}\text { Não-histerectomizadas } \\
\qquad(n=102)\end{array}$ & $\mathbf{P}$ \\
\hline Menarca (anos)* & $12,9 \pm 2,2$ & $13,3 \pm 1,6$ & 0,241 \\
\hline Paridade* & $3,8 \pm 2,6$ & $3,9 \pm 2,5$ & 0,961 \\
\hline Paridade (\% nulíparas) & 10,0 & 1,0 & 0,042 \\
\hline Paridade (\% multíparas) & 90,0 & 99,0 & \\
\hline Laqueadura tubária (\%) & 23,3 & 38,2 & 0,133 \\
\hline Tempo de amenorréia (anos)* & & $3,9 \pm 3,2$ & $\star \star 1$ \\
\hline Tempo de sintomas (anos)* & $4,2 \pm 4,5$ & & $\star \star 1$ \\
\hline Tempo de histerectomia (anos)* & $12,1 \pm 7,7$ & & \\
\hline Idade à histerectomia (anos)* & $41,0 \pm 7,3$ & & \\
\hline
\end{tabular}

* Média \pm desvio-padrão.

** Comparação entre o tempo de amenorréia do grupo não-histerectomizadas

e o tempo de sintomas climatéricos do grupo histerectomizadas, $p=0,32$.

1 Teste não paramétrico de Mann-Whitney para amostras independentes.

2 Teste exato de Fisher.

3 Teste qui-quadrado de Pearson. 
direta com a DMO enquanto que a paridade, uma associação inversa (Tabela 3 ).

Nessa regressão, o antecedente de histerectomia simples não evidenciou associação significativa, quando avaliado em conjunto com as demais variáveis consideradas.

A comparação das médias da DMO nos grupos pareados não mostrou diferenças significativas, tanto em nível do fêmur, como da coluna lombar (Tabela 4).

Na Tabela 5 os valores de T-score foram agrupados em normal, quando apresentavam valores superiores a -1DP e alterado, quando inferiores a -1DP. Não houve diferenças significativas na porcentagem de densitometrias normais e alteradas nos dois grupos, tanto nos três locais do fêmur, como na coluna lombar.

\section{Discussão}

Os resultados deste estudo mostraram que o antecedente de histerectomia com conservação ovariana bilateral, não se relacionou à densidade mineral óssea das mulheres, quando avaliadas na pós-menopausa. Mais ainda, não houve diferenças na massa óssea das mulheres histerectomizadas, quando comparadas a um grupo de mulheres também na pós-menopausa, sem o antecedente de histerectomia, pareadas por idade e índice de massa corporal.

Este resultado foi semelhante aos relatados por Modesto Filho et al. (1996), que verificaram que a histerectomia com conservação ovariana não proporcionou aumento da perda óssea, além do esperado para este período, e por Kritz-Silverstein \& Barret-Connor (1996), que avaliaram os efeitos tardios na massa óssea de mulheres histerectomizadas, sendo $182 \mathrm{com}$ conservação ovariana bilateral e 164 com ooforectomia bilateral concomitante. As mulheres apresentaram idade entre 60 e 89 anos, com média etária de 75 anos. Observou-se que não havia diferenças significativas entre os dois grupos, e concluiu-se que embora a ooforectomia bilateral cause perda da massa óssea mais rápido quando comparada ao grupo com conservação ovariana, a DMO final era semelhante entre os grupos.

Em Nova York, Hreshyshyn et al. (1988) avaliaram a massa óssea de 37 mulheres histerectomizadas e de 91 com menopausa natural, com idade entre 35 e 65 anos. A densidade mineral óssea do grupo das histerectomizadas foi significativamente menor que a do grupo com menopausa natural. Na Universidade Federal de São Paulo, realizou-se um estudo avaliando a densidade mineral óssea de 11 mulheres histerectomizadas e 11 não histerectomizadas, na

\begin{tabular}{|c|c|c|c|c|}
\hline \multicolumn{5}{|c|}{$\begin{array}{l}\text { Fatores associados à densidade mineral óssea do fêmur e da coluna lombar. } \\
\text { Regressão linear múltipla. }\end{array}$} \\
\hline Local & $\mathbf{r}^{2}$ & Variáveis & Coeficiente & $P$ \\
\hline \multirow[t]{2}{*}{ Colo } & 0,1836 & Idade 1 & $-0,0068$ & $<0,01$ \\
\hline & & IMC2 & 0,0108 & $<0,01$ \\
\hline \multirow[t]{2}{*}{ Wards } & 0,1008 & Idade & $-0,0081$ & $<0,01$ \\
\hline & & IMC & 0,0087 & 0,01 \\
\hline Trocânter & 0,1281 & IMC & 0,0125 & $<0,01$ \\
\hline \multirow[t]{5}{*}{$\mathrm{L}_{2}-\mathrm{L}_{4}$} & 0,2199 & Idade & $-0,0069$ & 0,01 \\
\hline & & IMC & 0,0094 & $<0,01$ \\
\hline & & Cor Parda & 0,1183 & $<0,01$ \\
\hline & & Escolaridade 1 & 2,519 & 0,01 \\
\hline & & Paridade & $-2,865$ & $<0,01$ \\
\hline
\end{tabular}

$r^{2}=$ coeficiente de determinação

1 em anos.

2 Índice de Massa Corporal em kg/m².

Tabela 4

Médias da densidade mineral óssea $\left(\mathrm{g} / \mathrm{cm}^{2}\right)$ do fêmur e da coluna lombar, segundo grupo de mulheres

histerectomizadas e não-histerectomizadas, pareadas por idade e índice de massa corporal.

\begin{tabular}{lccccc}
\hline \multirow{2}{*}{ Local } & \multicolumn{2}{c}{ Histerectomizadas $(\mathbf{n}=\mathbf{3 0})$} & \multicolumn{2}{c}{ Não-histerectomizadas $(\mathbf{n}=\mathbf{3 0})$} & D \\
& Média & DP & Média & DP \\
\hline Colo & 0,906 & 0,139 & 0,941 & 0,131 & $* 1$ \\
Wards & 0,772 & 0,171 & 0,804 & 0,143 & $* 1$ \\
Trocânter & 0,733 & 0,116 & 0,784 & 0,117 & $* 1$ \\
$\mathrm{~L}_{2}-\mathrm{L}_{4}$ & 1,098 & 0,144 & 1,081 & 0,162 & 0,782 \\
\hline
\end{tabular}

1 Comparação multivariada das médias dos três locais do fêmur; IC 95\% de Bonferroni; $p=0,11$.

2 Teste t de Student para amostras pareadas. 
Distribuição percentual das mulheres histerectomizadas e não-histerectomizadas, segundo categoria de T-score do fêmur e da coluna lombar, pareadas por idade e índice de massa corporal.

\begin{tabular}{lccccc}
\hline Local & \multicolumn{2}{c}{$\begin{array}{c}\text { Histerectomizadas }(\mathbf{n}=\mathbf{3 0}) \\
\text { Normal }\end{array}$} & Alterado & $\begin{array}{c}\text { Não-histerectomizadas }(\mathbf{n}=\mathbf{3 0}) \\
\text { Normal }\end{array}$ & $\begin{array}{c}\text { Alterado } \\
\text { Colo }\end{array}$ \\
Wards & 66,7 & 33,3 & 70,0 & 30,0 & 0.78 \\
Trocânter & 56,7 & 43,3 & 56,7 & 43,3 & 1,00 \\
$\mathrm{~L}_{2}-\mathrm{L}_{4}$ & 66,7 & 13,3 & 83,3 & 16,7 & 0,14 \\
\hline
\end{tabular}

Teste qui-quadrado de Pearson.

faixa etária entre 35 e 45 anos. O grupo de histerectomizadas apresentou DMO significativamente menor em nível do triângulo de Wards (Simões et al., 1995). Watson et al. (1995) avaliaram a DMO de quarenta mulheres menopausadas histerectomizadas com conservação ovariana e de quarenta mulheres com menopausa natural. O grupo de mulheres histerectomizadas apresentou densidade mineral óssea significativamente menor.

Resultados contraditórios foram observados com a publicação de Ravn et al. (1995) na Dinamarca. Estes pesquisadores analisaram a DMO de 69 mulheres histerectomizadas com conservação ovariana, e de 427 mulheres com menopausa natural. As mulheres histerectomizadas apresentavam maior densidade mineral óssea em nível do colo proximal, de cerca de $6 \%$ a $11 \%$, comparadas ao grupo controle.

Tuppurainen et al. (1995) realizaram na Finlândia um estudo de base populacional, selecionando aleatoriamente 1.605 mulheres, entre 47 e 56 anos. Dessas, 265 eram histerectomizadas com a conservação de um ou ambos os ovários. A realização da densitometria óssea nessas mulheres, mostrou que o grupo de histerectomizadas apresentou DMO maior, comparado ao grupo de referência. Os autores concluíram que no grupo de histerectomizadas, a miomatose uterina foi responsável por $43,8 \%$ das indicações cirúrgicas, que é uma patologia hiperestrogênica, mantendo assim a massa óssea.

O presente estudo difere dos citados porque os grupos foram controlados por características que poderiam influenciar o resultado final. Segundo Dean (1997), as pesquisas publicadas, apesar de controlarem as mulheres por idade, duração dos sintomas climatéricos e índice de massa corporal, falharam por não considerarem os hábitos e principalmente o passado reprodutivo das pacientes.

Cabe citar também, que a média etária das mulheres avaliadas neste estudo foi de aproxi- madamente 53 anos, sendo semelhante entre os grupos. A média etária à histerectomia foi de 41 anos, mas os sintomas climatéricos somente apareceram por volta dos 49 , idade essa compatível com a verificada em estudos que descreveram a idade de ocorrência da menopausa natural em nosso meio. Isso pode ser considerado indicativo de que a histerectomia, além de não ter alterado a massa óssea, não causou falência ovariana.

Em relação aos fatores reprodutivos, não observamos diferenças nas mulheres histerectomizadas em relação ao grupo de referência. Apesar da influência dessas variáveis sobre a massa óssea não ser claramente estabelecida, algumas adquirem especial importância pela sua alta prevalência. A laqueadura tubária, por exemplo, pode alterar o aporte sangüíneo ovariano, influenciando negativamente a massa óssea.

A paridade tem sido relatada por vários autores como um fator associado à idade, à menopausa e, conseqüentemente, às suas repercussões na massa óssea. As mulheres nulíparas têm menopausa mais precoce, enquanto o aumento da paridade correlaciona-se a uma menopausa mais tardia.

O índice de massa corporal apresenta uma correlação positiva com a massa óssea. Pessoas de maior peso corporal, apresentam uma absorção de cálcio mais eficiente e um aparato de remodelação óssea menos sensível ao paratormônio (PTH), conservando mais a massa óssea. Além disso, o peso corporal interage com os hormônios gonadais na determinação do pico de massa óssea, de modo que o excesso de peso protege contra os efeitos adversos da deficiência estrogênica sobre o esqueleto.

A escolaridade, por ser indicativa de modo indireto de fatores nutricionais, as mulheres com menor escolaridade teoricamente teriam menor nível sócio-econômico e ingestão de cálcio mais deficiente. Como é prioridade do or- 
ganismo manter os níveis sangüíneos de cálcio dentro dos limites constantes, sempre que as concentrações plasmáticas exigirem, essa substância será retirada do osso, resultando em uma deterioração da qualidade óssea.

Fatores genéticos parecem ser responsáveis pelas diferenças de massa óssea encontradas entre indivíduos de diferentes cores de pele. Os negros apresentam maior DMO e menor incidência de fraturas que indivíduos brancos e asiáticos. Acredita-se que apresentem mecanismos mais eficientes para a conservação do cálcio no organismo, em decorrência de níveis maiores de PTH e vitamina D.

O tabagismo está associado à redução do pico de massa óssea, antecipação da menopausa e ao baixo peso, todos considerados como fatores de risco para osteoporose.

No presente estudo, as variáveis IMC, escolaridade, cor de pele e tabagismo apresentaram médias muito semelhante entre os grupos histerectomizadas e "não-histerectomizadas" diminuindo assim os viéses de seleção, aumentando a fidelidade dos resultados.

Nas mulheres histerectomizadas, a confirmação da conservação ovariana bilateral baseou-se na informação da mulher ou na presença de laudo anatomopatológico referindo somente a retirada do útero. A ultra-sonografia não foi utilizada para a confirmação dos ovários, pois nas mulheres na pós-menopausa ocorre uma esperada atrofia gonadal, dificultando em muito a detecção ecográfica. Se tivéssemos incluindo neste estudo somente mulheres com ovários visíveis à ultra-sonografia, estaríamos cometendo um outro viés de seleção, pois estaríamos excluindo aquelas que apresentassem ovários atróficos.
Reconhecemos como a principal limitação na interpretação dos resultados deste estudo, o fato de não se tratar de um estudo prospectivo, com medidas da DMO antes e depois da histerectomia. Pode ser também que tenhamos avaliado o efeito final da histerectomia, pois conhecer o momento exato da falência ovariana nas mulheres histerectomizadas pode ser muito difícil, senão impossível, pela ausência do sinal objetivo, que é a amenorréia de 12 meses.

Em revisão da literatura, Dean (1997) analisou a ação da histerectomia sobre a DMO e concluiu que os achados dos estudos são inconsistentes e os resultados inconclusivos. A maioria dos estudos não apresentava controle das pacientes histerectomizadas e não histerectomizadas quanto aos fatores que poderiam influenciar a massa óssea, e o desenho de todos os estudos foram de corte transversal, como o atual. Essa pesquisadora inglesa está realizando um estudo de coorte longitudinal, com 44 mulheres histerectomizadas no menacme, com densitometria óssea prévia à cirurgia, e outra três anos após a histerectomia, além do estudo hormonal. Embora a pesquisa não tenha terminado, a autora publicou nessa revisão, que pelos seus resultados parciais, não parece haver diferenças entre as avaliações da massa óssea previamente à histerectomia e após três anos de seguimento. Os resultados que observamos no presente estudo são coerentes com as observações iniciais dessa autora. Entretanto, pelas controvérsias na literatura, recomendamos que essa questão, a diminuição na massa óssea pós-histerectomia, seja considerada e discutida com as pacientes previamente à cirurgia.

\section{Agradecimentos}

Este estudo recebeu apoio da Coordenação de Aperfeiçoamento de Pessoal de Nível Superior (CAPES). 


\section{Referências}

DEAN, S., 1997. Hysterectomy and bone mineral density. British Journal of Hospital Medicine, 57:207209.

HRESHCHYSHYN, M. M.; HOPKINS, A.; ZYLSTRA, S. \& ANBAR, M., 1988. Effects of natural menopause, hysterectomy and ooforectomy on lumbar spine and femural neck bone densities. Obstetrics and Gynecology, 72:631-638.

KAISER, R.; KUSCHE, M. \&WÜRZ, H., 1989. Hormone levels in women after hysterectomy. Archives of Gynecology and Obstetrics, 244:169-173.

KRITZ-SILVERSTEIN, D. \& BARRET-CONNOR, E., 1996. Oophorectomy status and bone density in older, hysterectomized women. American Journal of Preventive Medicine, 12:424-429.

LIMA, M. F. P.; REIS, R. M.; BONDUKI, C. E.; ZAMONER, M. R. F.; SILVA, C. S. \& SALUM, R., 1996. Análise da função ovariana em mulheres histerectomizadas. Revista Brasileira de Medicina Ginecologia e Obstetrícia, 7:203-206.

MODESTO FILHO, J.; LONDRE, S. J. B.; FERREIRA FILHO, J. A.; VASCONCELOS, H. C.; AZEVEDO, L. C. P. \& AZEVEDO, L. A. P., 1996. Efeito da ooforectomia e histerectomia sobre a densidade mineral óssea. Revista Brasileira de Ginecologia e Obstetrícia, 18:123-128.

O'DWYER, G., 1998. Avaliação da Qualidade da Assistência Prestada a Mulheres Submetidas à Histerectomia por Leiomioma. Dissertação de Mestrado, Rio de Janeiro: Instituto Fernandes Figueira, Fundação Oswaldo Cruz.

QUINN, A. J.; BARRETT, T.; KINGDOM, J. C. P. \& MURRAY, G. D., 1994. Relationship between hysterectomy and subsequent ovarian function in a district hospital population. Journal of Obstetrics and Gynaecology, 14:103-107.
RAVN, P.; LIND, C. \& NILAS, L., 1995. Lack of influence of simple premenopausal hysterectomy on bone mass and bone metabolism. American Journal of Obstetrics and Gynecology, 172:891-895.

SIDDLE, N.; SARREL, P. \& WHITEHEAD, M., 1987. The effect of hysterectomy on the age of ovarian failure: Identification of woman with premature loss of ovarian function and literature review. Fertility and Sterility, 47:94-100.

SIMÕES, R. D.; BARACAT, E. C.; SZEJNFELD, V. L.; LIMA, G. R.; GONÇALVES, W. J. \& BORTOLETTO, C. C., 1995. Effects of simple hysterectomy on bone loss. São Paulo Medical Journal, 113:1012-1016.

SOUZA, A. Z.; FONSECA, A. M.; IZZO, V. M.; CLAUZE, R. M. \& SALVATORE, C. A., 1986. Ovarian hystology and function after total abdominal hysterectomy. Obstetrics and Gynecology, 68:847-849.

TUPPURAINEN, M.; KRÖGER, H.; SAARIKOSKI, S.; HONKANEN, R. \& ALHAVA, E., 1995. The effects of gynecological risk factors on lumbar and femoral bone mineral density in peri- and postmenopausal women. Maturitas, 21:137-145.

WATSON, N. R.; STUDD, J. W. W.; GARNETT, T. \& MILLIGAN, P., 1995. Bone loss after hysterectomy with ovarian conservation. Obstetrics and Gynecology, 86:72-77.

WILCOX, L. S.; KOONIN, L. M.; POKRAS, R.; STRAUSS, L. T.; XIA, Z. \& PETERSON, H. B., 1994. Hysterectomy in tue United States, 1988-1990. Obstetrics and Gynecology, 83:549-555.

Recebido em 17 de maio de 2000

Versão final reapresentada em 1 de março de 2002

Aprovado em 22 de maio de 2002 